\title{
EXISTENCE OF POSITIVE SOLUTIONS DUE TO NON-LOCAL INTERACTIONS IN A CLASS OF NONLINEAR BOUNDARY VALUE PROBLEMS*
}

\author{
FORDYCE A. DAVIDSON ${ }^{\dagger}$ AND NIALL DODDS D $^{\ddagger}$
}

\begin{abstract}
We consider a class of non-local boundary value problems of the type used to model a variety of physical and biological processes, from Ohmic heating to population dynamics. Of particular relevance therefore is the existence of positive solutions. We are interested in the existence of such solutions that arise as a direct consequence of the non-local interactions in the problem. Conditions are therefore imposed that preclude the existence of a positive solution for the related local problem. Under these conditions, we prove that there exists a unique positive solution to the boundary value problem for all sufficiently strong non-local interactions and no positive solutions exists otherwise.
\end{abstract}

Key words. non-local, positive solutions, bifurcation

AMS subject classifications. 35J25; 35B32

1. Introduction. In this paper we study the steady states of a class of non-local differential equations of the form

$$
\begin{gathered}
u_{t}(x, t)=\sum_{i=1}^{n}\left(a_{i}(x) u_{x_{i}}(x, t)\right)_{x_{i}}+b(x) u(x, t)+\epsilon \tilde{u}(t)+n(x, \epsilon, u(x, t), \tilde{u}(t)) \quad x \in U, t>0 \\
u(x, t)=0 \quad \text { for } x \text { on } \partial U, t \geq 0 ; \quad u(x, 0)=u_{0}(x)
\end{gathered}
$$

where $U$ is a bounded connected subset of $\mathbb{R}^{n}, n=1,2,3$ with a suitably smooth boundary, $\partial U, \epsilon \in \mathbb{R}^{+}, \tilde{u}(t):=\int_{U} u(x, t) d x$, and $n: U \times \mathbb{R} \times \mathbb{R} \times \mathbb{R} \rightarrow \mathbb{R}$ with $n(x, \epsilon, \zeta, \tilde{\zeta})=o(\zeta)$ for $\zeta$ near zero, uniformly in bounded $\epsilon$ intervals. Moreover, we assume that $n(x, 0, u, \tilde{u})=f(x, u)$ for some suitable function $f$.

Equation (1) is referred to as a non-local differential equation, as it contains the functional $\tilde{u}$, the value of which depends upon the value of $u(x)$ the domain $U$. The parameter $\epsilon$ can be considered as a measure of the strength of the non-local effects in the equation: for $\epsilon=0$, the final condition on $n$ given above, ensures that (1) reduces to a local differential equation.

Non-local equations of the type given in (1) arise in the study of a wide variety of applications, including Ohmic heating [21, 28, 30], the formation of shear bands in materials [2], heat transfer in thermistors [19], combustion theory [32], the electric ballast resistor [8], microwave heating of ceramic materials [6, 29], and population dynamics [25]. An extensive survey of results, techniques and applications of nonlocal reaction-diffusion equations of the form studied here is given in [22].

Steady state solutions of (1) must clearly satisfy the system

$$
\begin{gathered}
\sum_{i=1}^{n}\left(a_{i}(x) u_{x_{i}}(x)\right)_{x_{i}}+b(x) u+\epsilon \tilde{u}+n(x, \epsilon, u(x), \tilde{u})=0, \quad x \in U ; \\
u(x)=0 \quad \text { for } x \text { on } \partial U
\end{gathered}
$$

\footnotetext{
*Received December 7, 2006; accepted for publication October 18, 2007.

${ }^{\dagger}$ Division of Mathematics, University of Dundee, Dundee, DD1 4HN, United Kingdom (fdavidso@ maths.dundee.ac.uk), tel. +44 1382 384692, fax +44 1382 385516. Author for Correspondence.

${ }^{\ddagger}$ Division of Mathematics, University of Dundee, Dundee, DD1 4HN, United Kingdom (ndodds@ maths.dundee.ac.uk), tel. +44 1382 384471, fax +44 1382385516 .
} 
Our aim in this paper is to establish the existence of positive solutions to (2). As we are interested in the existence of positive solutions to (2) that arise as a direct consequence of the non-local interactions in the equation, conditions will be imposed that preclude the existence of a positive solution for $\epsilon=0$, i.e. in the local case.

Existence of positive steady state solutions of non-local differential equations has been studied previously by many authors (e.g. [1, 6, 8, 9, 21, 23, 25, 29]). However, the effect of the non-local terms, in isolation, on the existence of positive solutions been not been previously considered.

The conditions on $n$ given above ensure that (2) has the trivial solution $u \equiv 0$ for all values of the strength parameter $\epsilon$. We will show that on increasing $\epsilon$ through a critical value, a continuum of positive steady state solutions bifurcates from this trivial solution branch. The behaviour of this continuum is investigated and conditions are given under which it can be guaranteed that (2) has a unique positive solution for all values of the strength parameter above the critical value. In order to best illustrate these results, we will first formulate them by considering a specific example, which arises in the study of population dynamics. These results are of interest in their own right but we will also return to the more generic form given in (2) and give conditions under which the various result continue to hold. The specific example we will consider is related to a class of local problems said to be of diffusive-Fisher-type:

$$
u_{t}(x, t)=\Delta u(x, t)+u(x, t) b(x)-u(x, t) f(x, u(x, t)) \quad x \in U, t>0,
$$

where $f$ is normally assumed to at most quadratic. System (3), with $b(x) \equiv b_{0}$ and $f(u)=u / K$ for constants $b_{0}$ and $K$ was first proposed by Fisher as a deterministic approximation to a stochastic model for the advancement of an advantageous gene through a population. System (3) is also a natural spatial extension of the logistic equation of population dynamics and and its use is commonplace in mathematical biology as a prototype model (see e.g. [7] and [18]). We will consider the steady states of a non-local extension of this model as given by (2) with

$$
n(x, \epsilon, u, \tilde{u})=-u\left[\rho u+\beta u^{2}+\gamma \epsilon \tilde{u}+\delta \epsilon \tilde{u}^{2}\right], \quad \rho, \gamma>0, \beta, \delta \geq 0,
$$

where $\rho, \beta, \gamma$ and $\delta$ are continuously differentiable, real-valued functions on $U$. From hereon we assume that the conditions given in (4) are satisfied.

The inclusion of the linear and nonlinear non-local terms in (2) represent, respectively, a contribution to the net birth rate (through e.g. dispersal of seeds or spores) and additional competition effects for a common resource such as water. For the derivation and analysis of a similar model see [25], where non-local effects are assumed to only contribute to the competition terms and the focus is on changes in system behaviour with domain size.

The approach we take is to rewrite (2) as an operator equation between appropriate function spaces. In this way, solutions to (2) can be shown to be equivalent to solutions of an associated nonlinear eigenvalue problem.

In Section 2 we precisely define the operator formulation of the problem and present some results regarding these non-local operators and relevant spectral properties. The main results of this paper are presented in Sections 3, 4 and 5. Sections 3 and 4 consider (2) when $n$ is of the form given in (4). In Section 3 the existence of non-trivial solutions to (2) is established using bifurcation theory, and in Section 4, by first developing appropriate a priori bounds, it is shown via the implicit function theorem that a unique positive solution of (2) exists for all values of the parameter $\epsilon$ above a critical value, whilst no positive solution exists for $\epsilon$ less than this value. In 
Section 5 we discuss the stability of positive solutions. Finally, in Section 6 we conclude by discussing how our results can be generalized to a wider class of non-local problems and briefly discuss some qualitative properties and open questions.

2. Preliminaries. Let $U$ be a bounded connected subset of $\mathbb{R}^{n}$, with boundary $\partial U \in C^{k+1}$, where $k=\left\lfloor\frac{n+4}{2}\right\rfloor$, and define

$$
\mathfrak{X}:=\left\{u \in C^{2, \alpha}(\bar{U}) \mid u=0 \text { on } \partial U\right\},
$$

for some $\alpha>0$. Then define the operators $A, L(\epsilon): \mathfrak{X} \rightarrow C^{\alpha}(\bar{U}), B: \mathfrak{X} \rightarrow \mathbb{R}$ by

$$
\begin{gathered}
{[A u](x)=\sum_{i=1}^{n}\left(a_{i}(x) u_{x_{i}}(x)\right)_{x_{i}}+b(x) u(x), \quad B u=\int_{U} u(x) d x,} \\
\text { and } L(\epsilon)=A+\epsilon B,
\end{gathered}
$$

where $a_{i}, b \in C^{k}(\bar{U})$ and $\epsilon \in \mathbb{R}$. We assume throughout that $-A$ is uniformly elliptic. We will also assume that $-A$ is positive definite.

Let $N: \mathbb{R} \times C^{\alpha}(\bar{U}) \rightarrow C^{\alpha}(\bar{U})$ be defined by

$$
[N(\epsilon, u)](x):=n(x, \epsilon, u(x), \tilde{u})
$$

where $n: U \times \mathbb{R} \times \mathbb{R} \times \mathbb{R} \rightarrow \mathbb{R}$ is defined in (4) above. The equation that we will consider throughout this paper is

$$
F(\epsilon, u):=L(\epsilon) u+N(\epsilon, u)=0 .
$$

Note that standard regularity arguments, the choice of $k$ and the conditions on the coefficients and on the domain $U$ are sufficient to ensure that $u \in \mathfrak{X}$ is a solution of (6) iff $u(x)$ satisfies the boundary value problem (2). Henceforth we shall therefore only refer to equation (6).

By (4), it follows that $N(\epsilon, u)=o\left(|u|_{\alpha}\right)$ as $|u|_{\alpha} \rightarrow 0$ uniformly in bounded $\epsilon$ intervals and that $u \equiv 0$ is a solution of $(6)$ for all $\epsilon \in \mathbb{R}$. Here $|u|_{\alpha}$ is the standard norm in $C^{\alpha}(\bar{U})$. Moreover, $L(\epsilon) u=0$ is the linearization of (6) about the trivial solution of $(6)$.

The operator $L(\epsilon)$ is closable by extension to an operator $\tilde{L}(\epsilon): H^{2}(U) \cap H_{0}^{1}(U) \subset$ $L^{2}(U) \rightarrow L^{2}(U)$. The spectral properties of this non-local operator have been extensively studied in certain cases: see $[4,5,14,20]$ for the case $n=1$; [23] for $n \geq 3$, and; $[1,15,16,24]$ for general $n$. It is shown in these papers that the eigenvalues, corresponding eigenfunctions and other spectral properties of the operator $L(\epsilon)$ are precisely those of its closed extension $\tilde{L}(\epsilon)$ and hence in the following, we simply refer to the operator $L(\epsilon)$ as defined in $(5)$.

When $\epsilon=0, L(\epsilon)$ reduces to the local operator $A$. With the above assumptions on $A$ in place, the spectral properties of this operator are well-known. The spectrum of $A$ consists entirely of isolated eigenvalues of finite multiplicity, which form a decreasing sequence of real numbers, tending to $-\infty$. The largest eigenvalue of $A$ is simple, is negative as $-A$ is positive definite, and has a corresponding eigenfunction, which is positive on $U$. Furthermore this is the only eigenvalue of $A$ to which there corresponds a positive eigenfunction. We denote the eigenvalues of $A$ by $\gamma_{k}, \forall k \in \mathbb{N}$, such that $0>\gamma_{1}>\gamma_{2} \geq \ldots \geq \gamma_{k} \geq \gamma_{k+1} \geq \ldots$ The general approach to establishing properties of the nonlocal operator $L(\epsilon)$ is to view $\epsilon$ as a homotopy parameter and use the 
perturbation theory of Kato [27]. In particular it can be shown that the spectrum of $L(\epsilon)$ consists of entirely of isolated eigenvalues $\left\{\lambda_{j}(\epsilon)\right\}_{j=1}^{\infty}$, which are continuous functions of $\epsilon$ and for which $\lambda_{k}(0)=\gamma_{k}$ (when indexed appropriately). It can be shown that under certain conditions, $L(\epsilon)$ has a principal eigenvalue, i.e. an eigenvalue for which corresponding eigenfunctions can be chosen to be positive. See $[14,15,16]$ for spectral results most relevant to the context here and also [20,24] for the general background to the spectral theory of these operators. Note that such results are not restricted to small $\epsilon$.

For the proofs of the following results and the discussion on generalizations, which follows, it is important to note that condition (4) and $u \geq 0$ on $U$ imply

$$
\begin{gathered}
x^{*} \in \bar{U}, u\left(x^{*}\right)=0 \Longrightarrow[N(\epsilon, u)]\left(x^{*}\right)=0 ; \\
x^{*} \in U, u\left(x^{*}\right)>0, \Longrightarrow[N(0, u)]\left(x^{*}\right)<0 ; \\
{[N(\epsilon, u)](x) v(x) \geq\left[N_{u}(\epsilon, u) v\right](x) u(x), \quad x \in U, \forall u, v \in \mathfrak{X}, u, v>0, \epsilon \geq 0 .}
\end{gathered}
$$

Remark 2.1. (a) For general non-local operators $N$, the growth condition $N(u)=$ $o\left(|u|_{\alpha}\right)$ does not necessarily imply the pointwise condition given in (7). For example consider the function $N(u)=-\tilde{u}^{2}$. Here, the growth condition holds but $N(u)=0$ iff $u \equiv 0$.

(b) As will be shown below, $-A$ positive definite and condition (8) together are sufficient to ensure that the local problem has no positive solutions.

(c) The right hand side of (9) may at first sight look somewhat unusual. Recall that for any suitable function $\phi, N_{u}(\epsilon, \phi)$. is a linear operator and hence $N_{u}(\epsilon, \phi) \psi$ is its action on the function $\psi$, which is itself a function. On setting $u=\phi$ and $v=\psi$, it is the pointwise multiplication of this function with $u$ that forms the right hand side of the inequality. As discussed in the final section, condition (9) is similar to a corresponding condition required for the existence of curves of positive solutions in local problems.

\section{Existence of non-trivial solutions.}

3.1. Bifurcation and local existence. We are interested in positive solutions of (6). To establish the existence of such solutions, we seek branches of positive solutions of (6) that bifurcate from the trivial solution branch. More specifically, we shall initially appeal to the classical local bifurcation result of Crandall and Rabinowitz [10] referred to as bifurcation from a simple eigenvalue.

By assumption, $F(\epsilon, u)=0$ has a branch of trivial solutions $(\epsilon, 0) \in \mathbb{R} \times \mathfrak{X}$. As a consequence of the implicit function theorem, bifurcation from this trivial branch can only occur at points where $F_{u}(\epsilon, 0)=L(\epsilon)$ is singular. A value $\epsilon^{*}$ for which this occurs, i.e. zero is a eigenvalue of $L\left(\epsilon^{*}\right)$, is defined to be a singular value of $L(\epsilon)$. Let the set of singular values of $L(\epsilon)$ be denoted by $S(L)$. It is clear from this definition that $S(L)$ consists of isolated points. Suppose now that there exists an $\epsilon^{*} \in S(L)$ such that zero is a simple eigenvalue of $L\left(\epsilon^{*}\right)$, i.e. $\operatorname{dim} N\left(L\left(\epsilon^{*}\right)\right)=1$ with $N\left(L\left(\epsilon^{*}\right)\right)=\operatorname{span}\left\{u^{*}\right\}$, say, and $u^{*} \notin R\left(L\left(\epsilon^{*}\right)\right)$. Suppose also that

$$
B u^{*} \notin R\left(L\left(\epsilon^{*}\right)\right) .
$$

Then we shall call $\epsilon^{*}$ a generic singular value of $L(\epsilon)$. 
Remark 3.1. If $L^{\prime}\left(\epsilon^{*}\right)$ denotes the derivative of $L(\epsilon)$ with respect to $\epsilon$ evaluated at $\epsilon=\epsilon^{*}$, then it follows from (5) that $L^{\prime}\left(\epsilon^{*}\right)=B$. Hence, our definition of a generic singular value coincides with, for example, a simple eigenvalue of multiplicity 1 [31] (and many subsequent, equivalent definitions).

Lemma 3.2. $L(\epsilon)$ has a unique singular value $\epsilon^{*}>0$. Moreover, $\epsilon^{*}$ is a generic singular value of $L(\epsilon)$. The eigenfunction $u^{*}$ corresponding to the zero eigenvalue of $L\left(\epsilon^{*}\right)$ can be chosen positive in $U$.

Proof. It is straightforward to show that condition (10) is in fact equivalent to $\lambda^{\prime}(\epsilon) \neq 0$, i.e. an eigenvalue $\lambda(\epsilon)$ of $L(\epsilon)$ passing through zero with non-zero speed as $\epsilon$ passes through $\epsilon^{*}$. It follows directly by results in [15] that a generic singular value, $\epsilon^{*}$, of $L(\epsilon)$ exists and is unique and that $L\left(\epsilon^{*}\right)$ has a positive eigenfunction corresponding to the zero eigenvalue. $\mathrm{Q}$

Lemma 3.2 ensures that on the trivial branch, the unique candidate for a bifurcation point is given by $\epsilon=\epsilon^{*}$, the generic singular value of $L(\epsilon)$. In fact,

TheOREM 3.3. $\left(\epsilon^{*}, 0\right) \in \mathbb{R} \times \mathfrak{X}$ is a bifurcation point of (6), and the bifurcation branch emanating from $\left(\epsilon^{*}, 0\right)$ has the following properties. There exists a positive real number, $s_{0}$, and continuous functions, $\phi:\left(-s_{0}, s_{0}\right) \rightarrow \mathbb{R}$ and $\psi:\left(-s_{0}, s_{0}\right) \rightarrow \mathfrak{X}$ with $\phi(0)=\psi(0)=0$ such that

$$
\left(\epsilon^{*}+\phi(s), s u^{*}+s \psi(s)\right)
$$

solves (6) $\forall s \in\left(-s_{0}, s_{0}\right)$. Furthermore, there exists a neighbourhood of $\left(\epsilon^{*}, 0\right) \in$ $\mathbb{R} \times \mathfrak{X}, \mathfrak{M}$ say, such that all non-trivial solutions of (6) in $\mathfrak{M}$ are given by (11).

Remark 3.4. By Lemma 3.2, the eigenfunction $u^{*}$ is positive. Clearly then the solutions given by (11) are positive for $s$ sufficiently small and positive.

Proof of Theorem 3.3. The proof follows directly from Theorem 1.7 in [10], under the hypotheses given in Section 2. In particular, as $A$ is a Fredholm operator of index zero and $B$ is a compact operator, $L(\epsilon)$ is also a Fredholm operator of index zero. Also, the transversality condition (condition (d) in Theorem 1.7 in [10]) is equivalent to our condition (10).

3.2. Global continua of non-trivial solutions. It is natural to ask to what extent the local branch defined above can be extended outwith the small neighbourhood, $\mathfrak{M}$. The global existence of branches of non-trivial solutions can be investigated via topological methods: central to this is the well-known global bifurcation theorem of Rabinowitz [33]. However, due to the formulation of the problem under discussion here, it will be necessary to use a slight modification of this result, (see e.g. [13] and the references therein; see also [3] for a partially similar result).

The global theory relies on certain compactness properties and therefore we must first reformulate our problem as follows.

First, we note that (6) is equivalent to

$$
(L(\epsilon)+k I) u=k I u-N(\epsilon, u),
$$

for any constant $k$. It can be assumed that $k$ is chosen such that $(L(\epsilon)+k I)^{-1}$ : $C^{\alpha}(\bar{U}) \rightarrow C^{\alpha}(\bar{U})$ exists, and hence (12) is equivalent to

$$
u=(L(\epsilon)+k I)^{-1}(k I u-N(\epsilon, u)) .
$$


i.e.

$$
u=T(\epsilon) u+H(\epsilon, u),
$$

where $T(\epsilon):=(L(\epsilon)+k I)^{-1} k I$ and $H(\epsilon, u):=-(L(\epsilon)+k I)^{-1} N(\epsilon, u)$. It follows from [24] that $(L(\epsilon)+k I)^{-1}: C^{\alpha}(\bar{U}) \rightarrow C^{\alpha}(\bar{U})$ is compact, therefore by standard arguments $T(\epsilon) \cdot+H(\epsilon, \cdot): C^{\alpha}(\bar{U}) \rightarrow C^{\alpha}(\bar{U})$ is compact, and any solution $(\epsilon, u) \in \mathbb{R} \times C^{\alpha}(\bar{U})$ of (14) provides a solution $(\epsilon, u) \in \mathbb{R} \times \mathfrak{X}$ of $(6)$.

LEMma 3.5. $\epsilon^{*}$ is a generic singular value of $I-T(\epsilon)$ iff $\epsilon^{*}$ is a generic singular value of $L(\epsilon)$.

Proof. It is straightforward to show that $\lambda(\epsilon)$ is an eigenvalue of $L(\epsilon)$ iff $\mu(\epsilon):=$ $\lambda(\epsilon) /(k+\lambda(\epsilon))$ is an eigenvalue of $I-T(\epsilon)$ and that $\mu(\epsilon)$ is a simple eigenvalue of $I-T(\epsilon)$ iff $\lambda(\epsilon)$ is a simple eigenvalue of $L(\epsilon)$. Suppose that $\epsilon^{*}$ is a generic singular value of $L(\epsilon)$. Then this implies $\lambda^{\prime}\left(\epsilon^{*}\right) \neq 0$. But then, $\mu^{\prime}\left(\epsilon^{*}\right) \neq 0$ and the result is proved. $\square$

Next, let $\Phi: \mathbb{R} \times C^{\alpha}(\bar{U}) \rightarrow C^{\alpha}(\bar{U})$ be defined by $\Phi(\epsilon, u)=u-T(\epsilon) u-H(\epsilon, u)$. Then it follows that $\Phi$ is a compact continuous perturbation of the identity and the Frèchet derivative of $\Phi$ with respect to $u$ at $(\epsilon, 0)$ is $I-T(\epsilon)$. For fixed $\epsilon, \Phi(\epsilon, u)$ is therefore of an appropriate form for the use of the Leray-Schauder degree. From the arguments above, it follows that $u \equiv 0$ is an isolated fixed point of $\Phi(\epsilon, u) \forall \epsilon \neq \epsilon^{*}$. Therefore for each fixed $\epsilon \neq \epsilon^{*}$, the degree of $\Phi$ with respect to the fixed point $u \equiv 0$ is given by

$$
\operatorname{deg}(\Phi(\epsilon, \cdot), 0)=\operatorname{deg}(I-T(\epsilon), 0)=(-1)^{\beta},
$$

where $\beta$ is the sum of the algebraic multiplicities of the eigenvalues $\mu(\epsilon)$ of $T(\epsilon)$ that are greater than 1 (see e.g. Chpt. 14 in [35]). By Lemma 3.5, $\epsilon^{*}$ is a unique generic singular value of $I-T(\epsilon)$ and it follows by the definition of the degree given in (15) that:

Lemma 3.6. Let $\epsilon_{1}<\epsilon^{*}<\epsilon_{2}$. Then

$$
\operatorname{deg}\left(\Phi\left(\epsilon_{1}, \cdot\right), 0\right)=-\operatorname{deg}\left(\Phi\left(\epsilon_{2}, \cdot\right), 0\right) \neq 0 .
$$

Finally, define the cones $\mathcal{P}^{ \pm}$by

$$
\mathcal{P}^{ \pm}=\operatorname{int}\{(\epsilon, u) \in \mathbb{R} \times \mathfrak{X}: \epsilon>0 \text { and } \pm u(x)>0 \forall x \in U\} .
$$

Remark 3.7. Note that no general maximum principle exists for non-local equations of the type studied here, and therefore it is possible, a priori, that non-negative solutions to (6) lie in $\partial \mathcal{P}^{+}$.

We can now obtain our main result of this section.

TheOrem 3.8. There exist unbounded continua $\mathcal{C}^{ \pm} \subset \mathbb{R} \times \mathfrak{X}$ of solutions of (6) such that $\left(\epsilon^{*}, 0\right) \in \mathcal{C}^{ \pm}$. Moreover,

$$
\left(\mathcal{C}^{ \pm} \backslash\left\{\left(\epsilon^{*}, 0\right)\right\}\right) \cap \mathcal{B}_{\eta}\left(\epsilon^{*}\right) \subset \mathbb{R} \times \mathcal{P}^{ \pm},
$$

where $\mathcal{B}_{\eta}\left(\epsilon^{*}\right)$ is the ball centre $\left(\epsilon^{*}, 0\right)$, radius $\eta$, and $\eta>0$ is sufficiently small. 
Proof. The proof follows closely that of Theorem 2.3 in [33]. By the definitions given above, $H(\epsilon, u)=o\left(|u|_{\alpha}\right)$ for $u$ near zero, uniformly on bounded $\epsilon$-intervals. Hence (14) is of the form discussed in [3] and [13] (these transpose the classical results of [33] to equations of the form given in (14)). The key criterion that the index changes as $\epsilon$ passes through $\epsilon^{*}$ is ensured by Lemma 3.6. Hence there exists a continuum $\mathcal{C}$ of nontrivial solutions to (14), which emanates from the bifurcation point $\left(\epsilon^{*}, 0\right)$ and which either joins $\infty$ in $\mathbb{R} \times C^{\alpha}(\bar{U})$ or joins $(\hat{\epsilon}, 0)$ where $\hat{\epsilon}$ is another singular value of $I-T(\epsilon)$. The uniqueness of $\epsilon^{*}$ ensures that the first alternative must hold. Following [33], it can be shown that sufficiently close to the bifurcation point, the continuum $\mathcal{C}$ can be locally decomposed into two sub-continua $\mathcal{C}^{ \pm}$. Moreover, sufficiently close to the bifurcation point these sub-continua must coincide with the branch of solutions given by Theorem 3.3. As a consequence of (11), the solutions on these subcontinua have the required sign. Finally, following the arguments in [33] (see also the remarks in $[12,13]$ ), this local structure of the continuum of solutions ensures that the alternatives stated above for the continuum $\mathcal{C}$ can in fact be applied to each of the sub-continua $\mathcal{C}^{ \pm}$. Again, the uniqueness of $\epsilon^{*}$ ensures that the second alternative holds for each sub-continuum. The result follows by the equivalence of the solutions of (6) and (14) as stated above and denoting the corresponding solution components in $\mathbb{R} \times \mathfrak{X}$ again by $\mathcal{C}^{ \pm}$.

Whilst Theorem 3.8 identifies the existence of an unbounded continua of nontrivial solutions of (6), it gives only partial information concerning the structure of the subcomponents. Of particular interest here, is the component $\mathcal{C}^{+}$on which solutions are known to be positive, close to the bifurcation point. We now show that $\mathcal{C}^{+} \backslash\left\{\left(\epsilon^{*}, 0\right)\right\} \subset \mathcal{P}^{+}$, i.e. all solutions on $\mathcal{C}^{+} \backslash\left\{\left(\epsilon^{*}, 0\right)\right\}$ are positive in $U$.

Theorem 3.9. $\mathcal{C}^{+} \backslash\left\{\left(\epsilon^{*}, 0\right)\right\} \subset \mathcal{P}^{+}$.

Proof. By Theorem 3.8, $\mathcal{C}^{+} \backslash\left\{\left(\epsilon^{*}, 0\right)\right\} \subset \mathcal{P}^{+}$in a sufficiently small neighbourhood of $\left(\epsilon^{*}, 0\right)$. We now show that $\mathcal{C}^{+} \backslash\left\{\left(\epsilon^{*}, 0\right)\right\} \cap \partial \mathcal{P}^{+}=\emptyset$. Suppose that $\mathcal{C}^{+} \backslash\left\{\left(\epsilon^{*}, 0\right)\right\} \cap$ $\partial \mathcal{P}^{+} \neq \emptyset$, i.e. suppose that either (i) there exists a point $\left(\epsilon_{0}, u_{0}\right) \in \mathcal{C}^{+}$for which $\epsilon_{0}=0$ and $u_{0}(x) \geq 0$ for $x \in U$, or (ii) there exists a point $(\hat{\epsilon}, \hat{u}) \in \mathcal{C}^{+}$, such that $\hat{\epsilon}>0$ and $\hat{u}(x) \geq 0$ for $x \in U$, whilst $\exists \hat{x} \in \bar{U}$ such that

$$
\hat{u}(\hat{x})=\hat{u}_{x_{1}}(\hat{x})=\hat{u}_{x_{2}}(\hat{x})=\ldots=\hat{u}_{x_{n}}(\hat{x})=0 .
$$

First considering (i), we have

$$
\left[A u_{0}\right](x)+\left[N\left(0, u_{0}\right)\right](x)=0 .
$$

Multiplying (16) by $u_{0}$ and integrating gives

$$
\int_{U}\left[A u_{0}\right](x) u_{0}(x)+\left[N\left(0, u_{0}\right)\right](x) u_{0}(x) d x=0 .
$$

However, as $-A$ is positive definite and conditions (7) and (8) hold, we must have that $u_{0}(x) \equiv 0$. But then this implies that $(0,0)$ is a bifurcation point, which contradicts the uniqueness of $\epsilon^{*}$. Now considering (ii), we note that either $\hat{u} \equiv 0$ or $\hat{u} \not \equiv 0$. If $\hat{u} \equiv 0$, then $(\hat{\epsilon}, 0)$ must be a bifurcation point of $L(\epsilon)$ and by Theorem 3.8, $\hat{\epsilon}=\epsilon^{*}$. Hence now consider $\hat{u} \not \equiv 0$. Then it follows from standard arguments for uniformly elliptic operators that $[A u](\hat{x}) \geq 0$, and by $(7)$ that $[N(\hat{\epsilon}, \hat{u})](\hat{x})=0$. Hence, by $(6)$ $\hat{\epsilon} B u \leq 0$ and it follows directly that $\hat{\epsilon} \leq 0$, which is again a contradiction.

Remark 3.10. Part (i) of the proof of this result shows that for the conditions given here, no positive solution of the local problem $(\epsilon=0)$ exists. 


\section{Positive solutions.}

4.1. Existence of positive solutions $\forall \epsilon>\epsilon^{*}$. We now establish apriori bounds that will be used to show the existence of a positive solution to (6) $\forall \epsilon>\epsilon^{*}$.

Lemma 4.1. Any $(\epsilon, u) \in \mathcal{P}^{+}$which is a solution of (6) has

$$
|u|_{\mathfrak{X}} \leq \Gamma(\epsilon),
$$

where $\Gamma(\epsilon)$ is a positive, increasing function of $\epsilon$, and $\Gamma(\epsilon)<\infty$ for all $\epsilon<\infty$.

Proof. Let $(\epsilon, u) \in \mathcal{P}^{+}$be a solution of (6). Then multiplying (6) by $u$ and integrating over $U$ and recalling the definition of $n$ given in (4) yields

$$
\begin{aligned}
\int_{U}[A u+\epsilon B u](x) u(x) d x & -\int_{U} \rho(x) u(x)^{3} d x \\
& -\int_{U}\left(\beta(x) u(x)^{3}+\epsilon \gamma(x) u(x) \tilde{u}+\epsilon \delta(x) u(x) \tilde{u}^{2}\right) u(x) d x=0 .
\end{aligned}
$$

Hence,

$$
\int_{U}[-A u](x) u(x)+\rho(x) u(x)^{3} d x \leq \epsilon \int_{U}[B u](x) u(x) d x=\epsilon\left(\int_{U} u(x) d x\right)^{2} .
$$

Applying Hölder's inequality to the RHS gives

$$
\int_{U}[-A u](x) u(x)+\rho(x) u(x)^{3} d x \leq \epsilon K\left(\int_{U} u(x)^{3} d x\right)^{\frac{2}{3}},
$$

for some constant $K$ dependent only on $U$. Now, $-A$ is positive definite and hence it follows that,

$$
\int_{U} u(x)^{3} d x \leq \epsilon \frac{K}{\min _{\bar{U}} \rho(x)}\left(\int_{U} u(x)^{3} d x\right)^{\frac{2}{3}}
$$

and therefore from (18) and (19) we have

$$
\int_{U}[-A u](x) u(x)+u(x)^{3} d x \leq \epsilon^{3} M
$$

for some positive constant $M$. Thus it has been shown that $u$ is bounded in $W^{1,2}(U)$. For $n \leq 3, W^{1,2}(U)$ is continuously embedded in $L^{6}(U)$. It therefore follows that $u$ is bounded in $L^{6}(U)$. Hence by the hypotheses on $N$ and by (6), $A u$ is bounded in $L^{2}(U)$, and therefore $u$ is bounded in $W^{2,2}(U)$. Then by an appropriate Sobolev embedding, $u$ is bounded in $C^{\alpha}(\bar{U})$, where for $n=1, \alpha=\frac{3}{2}$, for $n=2, \alpha$ is any positive number less than 1 , and for $n=3, \alpha=\frac{1}{2}$ (see e.g. Theorem 6 in Section 5.6 of [17]). The bound in $\mathfrak{X}$ then follows from (6) and the fact that $L(\epsilon)$ is non-singular for all $\epsilon \neq \epsilon^{*}$. $\square$

THEOREM 4.2. There exists a positive solution to (6) for each $\epsilon>\epsilon^{*}$.

Proof. By Theorem 3.9, the continuum of positive solutions $\mathcal{C}^{+}$emanates from $\left(\epsilon^{*}, 0\right)$ and joins $\infty$ in $\mathbb{R} \times \mathfrak{X}$. Lemma 4.1 ensure that this occurs at $\epsilon=\infty$. The result then follows directly. 
4.2. Uniqueness of positive solutions. We now establish that the continuum $\mathcal{C}^{+}$is in fact a curve, emanating from the bifurcation point $\left(\epsilon^{*}, 0\right)$. This will be done by applying the implicit function theorem and hence it is required to show that $D_{u} F(\hat{\epsilon}, \hat{u}) \cdot: \mathfrak{X} \rightarrow C^{\alpha}(\bar{U})$ is bijective, provided $F(\hat{\epsilon}, \hat{u})=0, \hat{\epsilon}>0$, and $\hat{u}(x)>0 \forall x \in$ $U$. From the definition of $F$, it follows that

$$
D_{u} F(\hat{\epsilon}, \hat{u}) z=L(\hat{\epsilon}) z+N_{u}(\hat{\epsilon}, \hat{u}) z,
$$

It was noted above that $L(\epsilon): \mathfrak{X} \rightarrow C^{\alpha}(\bar{U})$ is a Fredholm operator of index zero for all $\epsilon \in \mathbb{R}$. Hence and similarly, it can be shown that $D_{u} F(\hat{\epsilon}, \hat{u}) \cdot \mathfrak{X} \rightarrow C^{\alpha}(\bar{U})$ is a Fredholm operator of index zero. Therefore $D_{u} F(\hat{\epsilon}, \hat{u})$ is bijective iff it is injective, i.e. $D_{u} F(\hat{\epsilon}, \hat{u}) z \equiv 0 \Leftrightarrow z \equiv 0$.

Remark 4.3. That the implicit function theorem can be applied to the example considered here, means that the previous global existence result is superseded. However, the implicit function theorem requires quite strict hypotheses to be placed on the linear operator $A$ and on $n$, e.g. of the type given in (4). The global results derived above hold for far weaker hypotheses and therefore will be of relevance to the more general problem discussed later.

LEMMA 4.4. The set $\mathcal{C}^{+}$is a $C^{1}$ parameterized curve $\epsilon \rightarrow(\epsilon, u(\epsilon))$ in $\mathbb{R} \times \mathfrak{X}$.

Proof. Let $(\hat{\epsilon}, \hat{u}) \in \mathcal{C}^{+}$and suppose that $D_{u} F(\hat{\epsilon}, \hat{u}) z=0$ for some $0 \not \equiv z \in \mathfrak{X}$. Multiplying (6) by $z$ and $(21)$ by $\hat{u}$, subtracting and then integrating over $U$, gives

$$
\begin{aligned}
& \int_{U}[A \hat{u}](x) z(x)-[A z](x) \hat{u}(x) d x \\
& +\int_{U}[\hat{\epsilon} B \hat{u}+N(\hat{\epsilon}, \hat{u})](x) z(x)-\left[\hat{\epsilon} B z+N_{u}(\hat{\epsilon}, \hat{u}) z\right](x) \hat{u}(x) d x \\
= & \int_{U}[N(\hat{\epsilon}, \hat{u})](x) z(x)-\left[N_{u}(\hat{\epsilon}, \hat{u}) z\right](x) \hat{u}(x) d x=0 .
\end{aligned}
$$

If $z$ is of one $\operatorname{sign}$ on $U$, then as $D_{u} F(\hat{\epsilon}, \hat{u}) z$ is linear in $z$, we can assume without loss of generality that $z(x)>0$ in $U$. But then (22) contradicts (9) and so $z$ must change sign on $U$. Without loss of generality, suppose then that $z(x)>0 \forall x \in U^{*} \subset U$, whilst $z(x)=0$ on $\partial U^{*}$. Hence, $z_{x_{i}} \nu^{i} \leq 0 \forall x \in \partial U^{*}, i=1, \ldots, n$ where $\nu=\left(\nu^{1}, \nu^{2}, \ldots, \nu^{n}\right)^{T}$ is the outward pointing unit normal vector on $\partial U^{*}$. By assumption, $-A$ is uniformly elliptic and therefore

$$
a_{i}(x) \geq 0 \quad \forall x \in \bar{U}, i=1, \ldots, n
$$

Therefore

$$
\int_{U^{*}}[A \hat{u}](x) z(x)-[A z](x) \hat{u}(x) d x=\int_{\partial U^{*}} \sum_{i=1}^{n} a_{i}(x)\left(\hat{u}_{x_{i}} z-z_{x_{i}} \hat{u}\right) \nu^{i} d S \geq 0,
$$

where $\int \cdot d S$ represents integration with respect to surface area. But on replacing $U$ by $U^{*}$ in (22) then (23) and (9) ensure a contradiction. Hence, $z \equiv 0$ and as by Theorem 3.9, $\mathcal{C}^{+} \backslash\left\{\left(\epsilon^{*}, 0\right)\right\} \subset \mathcal{P}^{+}$, we have just shown that $D_{u} F(\hat{\epsilon}, \hat{u}): \mathfrak{X} \rightarrow C^{\alpha}(\bar{U})$ is bijective at all points $(\hat{\epsilon}, \hat{u}) \in \mathcal{C}^{+} \backslash\left\{\left(\epsilon^{*}, 0\right)\right\}$. The result now follows by a standard application of the implicit function theorem.

Finally, we have our most complete result. 
THEOREM 4.5. Equation (6) has a unique positive solution for all $\epsilon>\epsilon^{*}$ and no positive solution for $0 \leq \epsilon \leq \epsilon^{*}$.

Proof. The previous results ensure that $\mathcal{C}^{+}$is a $C^{1}$ curve parameterised by $\epsilon$, that joins $\infty$ in $\mathcal{P}^{+}$at $\epsilon=\infty$.

Suppose now that $(\bar{\epsilon}, \bar{u}) \in \mathcal{P}^{+}$is a solution of $(6)$ not in $\mathcal{C}^{+}$. Then it follows directly from the proof of Lemma 4.4 that the implicit function theorem can be applied at $(\bar{\epsilon}, \bar{u})$. First suppose that $\bar{\epsilon} \in\left[\epsilon^{*}, \infty\right)$. Then a maximal $C^{1}$ curve $\overline{\mathcal{C}}^{+}$can be constructed of positive solutions to (6) passing through $(\bar{\epsilon}, \bar{u})$. Now, by Lemma 4.1 and following similar arguments to those in the proof of Theorem 3.9, it can be shown that this curve must also remain bounded in $\mathcal{P}^{+}$for $\epsilon<\infty$ and as $\epsilon^{*}$ is the unique singular value of $L(\epsilon)$, this curve must join $\left(\epsilon^{*}, 0\right)$ in $\mathbb{R} \times \mathfrak{X}$. But then by the uniqueness of the solution curve $\mathcal{C}^{+}$in any sufficiently small neighbourhood, it follows that $\overline{\mathcal{C}}^{+} \equiv \mathcal{C}^{+}$.

Finally, suppose that $\bar{\epsilon} \in\left(0, \epsilon^{*}\right]$. Then again applying the implicit function theorem at $(\bar{\epsilon}, \bar{u})$ and using similar arguments to those above, shows that no positive solution can exist for values of $\epsilon$ in this interval.

5. Stability. The solutions discussed so far arise as steady states of the initialboundary value problem (1). Therefore a relevant question to ask is: are these solutions stable to perturbation? (It is likely that only stable solutions would be observed in application.) Following the discussions above and standard arguments (see for example $[26,35])$, the linear, asymptotic stability of a solution $\hat{u}$ of (1) is determined by the eigenvalues of the linear operator $F_{u}(\epsilon, \hat{u})$. (Essentially, this follows from the equivalence of steady state solutions of the boundary value problem and $F(\epsilon, u)=0$ as discussed above, that the operator $F_{u}(\epsilon, \hat{u})$ generates an analytic semi-group and the compactness of the inverse of $F_{u}(\epsilon, \hat{u})$.) The stability result of Henry states that the solution $\hat{u}$ is linearly, asymptotically stable if $\operatorname{Re}(\mu)<0$ for all $\mu \in \sigma\left(F_{u}(\epsilon, \hat{u})\right)$ and is unstable otherwise.

First, we note that $F_{u}(\epsilon, 0)=L(\epsilon)$ is self-adjoint and has compact resolvent. Hence its spectrum is real and consists entirely of isolated eigenvalues. Next, by the definition of the unique singular value $\epsilon^{*}$ given above, it follows that all the eigenvalues of $F_{u}(\epsilon, 0)$ are negative for $\epsilon<\epsilon^{*}$ and that (at least) the simple, principal eigenvalue $\lambda_{p}(\epsilon)>0$ for $\epsilon>\epsilon^{*}$. Hence, the trivial solution $u=0$ is stable for $0 \leq \epsilon<\epsilon^{*}$ and unstable for $\epsilon>\epsilon^{*}$.

Next we consider the branch of positive solutions. In what follows, we assume that Theorem 4.5 holds and hence there exists a unique branch of positive solutions extending to $\epsilon=\infty$ as detailed there. In a neighbourhood of the bifurcation point, the branch of positive solutions is given by $\left(\epsilon^{*}+\phi(s), s u^{*}+s \psi(s)\right)$ for $s$ sufficiently small and positive. Hence the stability of positive solutions close to the bifurcation point is determined by the eigenvalues of the operator $F_{u}\left(\epsilon+\phi(s), s u^{*}+s \psi(s)\right)$. Note that this operator has a principal eigenvalue $\mu_{p}(s)$ for which $\mu_{p}(0)=0$ and all other eigenvalues $\mu(s)<0$, for $s$ sufficiently small (by continuity of the spectrum of $F_{u}$ and noting that, whilst $F_{u}$ may not be self-adjoint, it still had compact resolvent). It follows from Theorem 1.16 in [11], that if $\phi^{\prime}(s)>0$, then $\mu_{p}(s)<0$ for $s \in(0, \hat{s})$, for some sufficiently small $\hat{s}>0$. By similar arguments to those presented in Corollary 8.25 of [35], it can be shown that $\phi^{\prime}(s)>0$ for $s$ sufficiently small. Hence, positive solutions are stable provided they lie in a neighbourhood of the bifurcation point. Finally, we note that solutions on the global extension of this branch can become unstable only if $\operatorname{Re}\left(\mu_{p}\right)$ passes through zero. Recall we are assuming that the implicit function theorem can be applied at each point $(\epsilon, u)$ on the branch. Hence, if $\sigma\left(F_{u}(\epsilon, u)\right)$ 
remains real, then by invoking the continuity of the spectrum of $F_{u}$, this leads to a contradiction. Hence all solutions on the branch are stable. However, it is exactly the non-local components of $F_{u}(\epsilon, u)$ which prevent it from being self-adjoint in general (even in a formal sense) and hence the spectrum of $F_{u}$ is not necessarily real. In this case, a general stability result does not follow directly.

6. The general problem. The results as stated for the specific example considered above continue to hold for considerably more generalized problems. Essentially, all that is required of the non-linear term $N$ is for it to be no more than cubic in $u$ and $\tilde{u}$ and the derivative of $N / u$ with respect to $u$ to be negative. (This latter condition corresponds directly to a commonly used condition imposed to ensure the existence of curves of solutions in the local case, see e.g. [34] and the references therein.) To be more precise, suppose that condition (4) is replaced by

$$
n(x, \epsilon, u, \tilde{u}):=-u[f(x, u)+\epsilon g(x, \tilde{u})],
$$

where $f(x, \cdot): \mathbb{R} \rightarrow \mathbb{R}$ and $g(x, \cdot): \mathbb{R} \rightarrow \mathbb{R}$ are strictly increasing functions satisfying

$$
K_{1} \zeta \leq f(x, \zeta) \leq K_{2} \zeta^{2}, \quad 0 \leq g(x, \zeta) \leq K_{3} \zeta^{2}
$$

for each $x \in U$ for some constants $K_{i}$, satisfying $0<K_{i} i=1,2,3$. Also, suppose that the derivatives $f^{\prime}(x, \cdot): \mathbb{R} \rightarrow \mathbb{R}$ and $g^{\prime}(x, \cdot): \mathbb{R} \rightarrow \mathbb{R}$ exist and are continuous. Then it is straightforward to show that conditions (7)-(9) still hold and hence all the results stated above hold in this more general case. (A little more working is required for the proof of Lemma 4.1, but the calculations take the same form.) It can be shown that the stability results in Section 5, also hold in this more general case.

For the bifurcation results in Section 3, the conditions on $N$ can be relaxed further still. Indeed, all that is required for Theorem 3.3 and Theorem 3.8 is that $N$ is sufficiently differentiable with respect to $\epsilon$ and $u$ and that condition (7) is satisfied. For Theorem 3.9, all that is required in addition is that condition (8) is satisfied.

The non-local term $\tilde{u}$ can also be replaced throughout with the more general expression

$$
[B u](x)=\tilde{u}(x):=c(x) \int_{U} c(x) u(x) d x
$$

with $c \in H^{k-1}(U), c(x)$ of one sign on $U$. This condition on $B$ is sufficient to ensure the existence of a unique positive generic singular value of $L(\epsilon)$, as defined above. Moreover, under these conditions, it can be shown that the eigenfunction corresponding to the zero eigenvalue of $L\left(\epsilon^{*}\right)$ is positive (see [15]). The specific form of $\tilde{u}$ is not otherwise required (apart from in the proof of Lemma 4.1, which again follows along very similar lines).

Finally, the specific form of the differential expression given in (2) is only required in the proof of Lemma 4.4. Therefore, all main results, other than Theorem 4.5, hold for more general differential expressions of the form

$$
\sum_{i, j=1}^{n}\left(a_{i j}(x) u_{x_{i}}(x)\right)_{x_{j}}
$$

where the operator $-A$, as defined in (5), is uniformly elliptic and positive definite.

We finish by stating that both general stability and qualitative properties of positive steady state solutions remain open questions. Numerical integration of the 
system (1) suggests that for simple forms of $n$ of the type given in (4), positive steady state solutions are stable to large perturbations and for a large range of $\epsilon$. Such solutions have a convex profile similar to related solutions of the diffusive Fisher equation. The profile is bounded and becomes increasing by flat in the interior of the domain and a steep boundary layer is formed as $\epsilon$ is increased. It is straight-forward to gain a heuristic understanding of this behaviour by simply dividing both sides of equation (2) by $\epsilon$ and assuming solutions remain convex and positive. However, it is exactly the lack of a general maximum principle for such non-local equations, which prevents this result from following directly. This merits further investigation.

\section{REFERENCES}

[1] W. Allegretto \& A. Barabanova, Positivity of Solutions of Elliptic Equations with Nonlocal Terms, Proc. Roy. Soc. Edinburgh Sect. A, 126 (1996), pp. 643-663.

[2] J. Bebernes, C. Li \& P. Talaga, Single Point Blowup for Nonlocal Parabolic Problems, Physica D, 134 (1999), pp. 48-60.

[3] J. Blat \& K.J. Brown, Global Bifurcation of Positive Solutions in some Systems of Elliptic Equations, SIAM J. Math. Anal., 17 (1986), pp. 1339-1353.

[4] A. Bose \& G.A. Kriegsmann, Stability of Localized Structures in Non-local Reaction-Diffusion Equations, Methods Appl. Anal., 5 (1998), pp. 351-366.

[5] A. Bose, A Geometric Approach to Singularly Perturbed Nonlocal Reaction-diffusion Equations, SIAM J. Math. Anal., 31 (2000), PP. 431-454.

[6] A. Bose \& G.A. Kriegsmann, Large Amplitude Solutions of Spatially Non-homogeneous Nonlocal Reaction Diffusion Equations, Methods Appl. Anal., 7 (2000), pp. 295-312.

[7] N.F. Britton, Reaction-diffusion Equations and their Applications to Biology, Academic Press, New York, 2000.

[8] N. ChafeE, The Electric Ballast Resistor: Homogeneous and Nonhomogeneous Equilibria, in Nonlinear Differential Equations: Invariance, Stability and Bifurcation (P. de Mottoni \& L. Salvadori eds.), 97-127, Academic Press, New York, 1981.

[9] F.J.S.A. CorrêA, S.D.B. Menezes \& J. Ferreira, On a Class of Problems Involving a Nonlocal Operator, Appl. Math. and Computation, 147 (2004), pp. 475-489.

[10] M.G. Crandall \& P.H. Rabinowitz, Bifurcation from Simple Eigenvalues, J. Funct. Anal., 8 (1971), pp. 321-340.

[11] M.G. Crandall \& P.H. Rabinowitz, Bifurcation, Perturbation of Simple Eigenvalues and Linearized Stability, Arch. Rat. Mech. Anal., 52 (1973), pp. 161-180.

[12] E.N. Dancer, On the Structure of Solutions of Non-linear Eigenvalue Problems, Ind. Univ. Maths. J., 23 (1974), pp. 1071-1076.

[13] F.A. Davidson, A Remark on the Global Structure of the Solution Set for a Generic Class of Non-linear Eigenvalue Problems, Comm. Appl. Anal., 6 (2002), pp. 449-454.

[14] F.A. Davidson \& N. Dodds, Spectral Properties of Non-local Differential Operators, Applicable Analysis, 85 (2006), pp. 717-734.

[15] F.A. Davidson \& N. Dodds, Spectral Properties of Non-local Uniformly Elliptic Operators, Elec. J. Diff. Eqns., 2006:126 (2006), pp. 1-15.

[16] N. Dodds, Non-local Differential Equations, Ph.D. Thesis, University of Dundee, (2005).

[17] L.C. Evans, Partial Differential Equations, American Mathematical Society, Providence, 1998.

[18] P. C. Fife, Mathematical aspects of reacting and diffusing systems, in Lecture Notes in Biomathematics, 28, Springer-Verlag, New York, 1979.

[19] A.C. Fowler, I.FrigaArd, \& S.D. Howison, Temperature Surges in Current Limiting Circuit Devices, SIAM J. Appl. Math., 52:4 (1992), pp. 998-1011.

[20] P. Freitas, A Nonlocal Sturm-Liouville Eigenvalue Problem, Proc. Roy. Soc. Edinburgh Sect. A, 124 (1994), pp. 169-188.

[21] P. Freitas \& M. Grinfeld, Stationary Solutions of an Equation Modeling Ohmic Heating, Appl. Math. Lett., 7 (1994), pp. 1-6.

[22] P. Freitas, Nonlocal Reaction-Diffusion Equations, Fields Institute Comms., 21 (1999), pp. 187-204.

[23] P. Freitas \& G. Sweers, Positivity Results for a Nonlocal Elliptic Equation, Proc. Roy. Soc. Edinburgh Sect. A, 128 (1998), pp. 697-715. 
[24] P. Freitas \& M.P. Vishnevskit, Stability of Stationary Solutions of Nonlocal ReactionDiffusion Equations in m-Dimensional Space, Diff. \& Int. Eqns., 13:1-3 (2000), pp. 265288.

[25] J. Furter \& M. Grinfeld, Local vs. Non-local Interactions in Population Dynamics, J. Math. Biol., 27 (1989), pp. 65-80.

[26] D. Henry, Geometric Theory of Semilinear Parabolic Equations, Springer-Verlag, Berlin, 1981.

[27] T. Kato, Perturbation Theory for Linear Operators, 2nd edition, Springer-Verlag, Berlin, 1976.

[28] N.I. Kavallaris, A.A. Lacey, \& D.E. Tzanetis, Global existence and divergence of critical solutions of a nonlocal parabolic problem in Ohmic heating process, Nonlinear Anal. TMA, 58 (2004), pp. 787-812.

[29] G.A. Kriegsmann, Hot Spot Formation in Microwave Heated Ceramic Fibres, IMA J. Appl. Math., 59 (1997), pp. 123-148.

[30] A.A. LACEY, Thermal runaway in a non-local problem modeling Ohmic heating: Part I, Euro. J. Appl. Math., 6 (1995), pp. 127-144.

[31] R.J. Magnus, A generalization of multiplicity and the problem of bifurcation, Proc. Lond. Math. Soc., 3 (1976), pp. 241-278.

[32] C.V. PAO, Blowing-up of Solution for a Nonlocal Reaction-Diffusion Problem in Combustion Theory, J. Math. Anal. Apps., 166 (1992), pp. 591-600.

[33] P.H. Rabinowitz, Some Global Results for Nonlinear Eigenvalue Problems, J. Funct. Anal., 7 (1971), pp. 487-513.

[34] B.P. RynNe, Solution curves of 2mth order boundary value problems, Electron. J. Differential Equations, 2004 (2004), pp. 1-16.

[35] E. Zeidler, Nonlinear Functional Analysis and its Applications, Vol. I - Fixed Point Theorems, Springer-Verlag, New York, 1986. 
F. A. DAVIDSON AND N. DODDS 